\title{
Stimulation of Deep Somatic Tissue with Capsaicin Produces Long-Lasting Mechanical Allodynia and Heat Hypoalgesia that Depends on Early Activation of the cAMP Pathway
}

\author{
K. A. Sluka \\ Graduate Program in Physical Therapy and Rehabilitation Science, Neuroscience Graduate Program, Pain Research \\ Program, University of lowa, lowa City, lowa 52242
}

Pain and hyperalgesia from deep somatic tissue (i.e., muscle and joint) are processed differently from that from skin. This study examined differences between deep and cutaneous tissue allodynia and the role of CAMP in associated behavioral changes. Capsaicin was injected into the plantar aspect of the skin, plantar muscles of the paw, or ankle joint, and responses to mechanical and heat stimuli were assessed until allodynia resolved. Capsaicin injected into skin resulted in a secondary mechanical allodynia and heat hypoalgesia lasting $\sim 3 \mathrm{hr}$. In contrast, capsaicin injection into muscle or joint resulted in a long-lasting bilateral (1-4 weeks) mechanical allodynia with a simultaneous unilateral heat hypoalgesia. The pattern and degree of inflammation were similar when capsaicin was injected into skin, muscle, or joint, with peak increases $24 \mathrm{hr}$ after injection. Heat hypoalgesia that occurs after injection into deep

Pain and hyperalgesia from deep somatic tissue, muscle, and joint are processed differently from that from skin (for review, see Mense, 1993; Schaible and Grubb, 1993). Pain from deep tissues is diff use, dull, aching, and difficult to localize, whereas that from cutaneous tissues is typically sharp and easy to localize (Kellgren, 1938; Ochoa and Torebjork, 1989; Simone et al., 1994; Marchettini et al., 1996). Furthermore, deep tissue pain is typically referred to superficial structures, whereas cutaneous pain is not usually referred (Ochoa and Torebjork, 1983; Marchettini et al., 1990). Longer-lasting enhancement of the ventral root reflex occurs after $\mathrm{C}$-fiber stimulation of a muscle nerve compared with C-fiber stimulation of a cutaneous nerve (Wall and Woolf, 1984). Afferent innervation of the spinal cord by skin and deep tissues is significantly different. Cutaneous nociceptors send dense projections to laminas I and II (Willis and Coggeshall, 1991). In contrast, joint and muscle afferents innervate laminas I and V, and there are no projections to lamina II (Craig et al., 1988; Mense and Craig, 1988). Furthermore, different biochemicals are observed in DRG that innervate the skin, muscle, and joint (O'Brien et al., 1989; Plenderleith and Snow, 1993). Thus, the same stimulus presented to muscle or joint would be expected

Received Jan. 24, 2002; revised March 8, 2002; accepted April 4, 2002.

This study was supported by National Institutes of Health Grants R01-NS-39734 and K02-AR-02201. I thank Tammy Lisi and Charles Cibula for excellent technical assistance.

Correspondence should be addressed to K. A. Sluka, Graduate Program in Physical Therapy and Rehabilitation Science, University of Iowa, Iowa City, IA 52242. E-mail: ksluka@blue.weeg.uiowa.edu.

Copyright (ㄷ) 2002 Society for Neuroscience $\quad 0270-6474 / 02 / 225687-07 \$ 15.00 / 0$ tissue was reversed by spinal blockade of adenylate cyclase or protein kinase A (PKA). Interestingly, mechanical allodynia was reversed if adenylate cyclase or PKA inhibitors were administered spinally $24 \mathrm{hr}$, but not 1 week, after injection of capsaicin. Spinally administered 8-bromo-cAMP resulted in a similar pattern, with heat hypoalgesia and mechanical allodynia occurring simultaneously. Thus, injection of capsaicin into deep tissues results in a longer-lasting mechanical allodynia and heat hypoalgesia compared with injection of capsaicin into skin. The mechanical allodynia depends on early activation of the cAMP pathway during the first $24 \mathrm{hr}$ but is independent of the cAMP pathway by 1 week after injection of capsaicin.

Key words: pain; analgesia; protein kinase A; adenylate cyclase; muscle; joint

behaviorally to result in a different patterned response than when injected into skin.

Capsaicin is a chemical irritant that causes a local neurogenic inflammation when injected into the skin. It produces primary heat and mechanical hyperalgesia and secondary mechanical hyperalgesia in humans and rats that resolves within hours (Simone et al., 1989; Lamotte et al., 1991; Sluka, 1997). There are also reports that capsaicin produces hypoalgesia in the area surrounding the injection site (Raja et al., 1984). Preliminary data show that injection of capsaicin into joint or muscle produces secondary mechanical allodynia and heat hypoalgesia (Sluka, 2000).

After intradermal capsaicin injection, spinothalamic tract cells show increased sensitivity to innocuous and noxious mechanical stimuli both at and distant from the site of injection (Simone et al., 1991; Sluka et al., 1997; Dougherty et al., 1998). However, the responsiveness of spinothalamic tract cells to heat is decreased after intradermal capsaicin injection (Sluka et al., 1997; Dougherty et al., 1998). Interestingly, the decreased responsiveness to heat of spinothalamic tract cells induced by intradermal capsaicin injection is reversed by inhibition of protein kinase A (PKA) (Sluka et al., 1997). Furthermore, sensitization of spinothalamic tract cells to mechanical stimuli (Sluka et al., 1997) and mechanical hyperalgesia (Sluka, 1997; Sluka and Willis, 1997) produced by intradermal injection of capsaicin are reversed by blockade of PKA or adenylate cyclase.

Thus, these experiments were undertaken to examine (1) differences in mechanical and heat sensitivity in response to capsaicin injection in the skin, muscle, and joint and (2) the role of the cAMP pathway in the behavioral responses to capsaicin injection. I hypothesized that capsaicin injection into muscle or joint would 
result in a longer-lasting allodynia than when injected into skin. I also hypothesized that heat hypoalgesia and mechanical allodynia induced by capsaicin injection would be reduced by spinal blockade of the cAMP pathway.

\section{MATERIALS AND METHODS}

All experiments were approved by the Animal Care and Use Committee at the University of Iowa and were in accordance with the National Institutes of Health and the International Association for the Study of Pain policies on use of laboratory animals.

Capsaicin injection. Male Sprague Dawley rats (250-350 gm; $n=173$; Harlan Sprague Dawley, Indianapolis, IN) were used for these experiments. Capsaicin $(0.2 \% ; 50 \mu \mathrm{l}$; Fluka Chemical Corporation, Milwaukee, WI) was injected (1) intradermally into the rostral aspect of the plantar surface of the paw, (2) intra-articularly into the ankle joint, or (3) intramuscularly into the plantar muscles of the foot. These tissues were chosen because they are all supplied by branches of the tibial nerve and they are in close proximity to each other (Greene, 1963). Capsaicin was dissolved in $10 \%$ Tween $80,10 \%$ ethanol, and sterile saline.

Myeloperoxidase assay. Myeloperoxidase is a neutrophil-specific marker used to assess the infiltration of neutrophils into an area of inflammation. To measure myeloperoxidase, a variation of the method described by Desser (1971) was used in which an enzyme unit is defined as the amount of enzyme necessary to produce an increase of 1 absorbance unit in 1 min. Rats were deeply anesthetized with sodium pentobarbital (Abbott Laboratories, North Chicago, IL) and guillotined, and tissues were removed promptly. The ankle joint, plantar muscles of the foot, or plantar surface of the skin was removed on both the ipsilateral and contralateral sides at $2 \mathrm{hr}(n=4), 24 \mathrm{hr}(n=4)$, and 1 week $(n=4)$ after capsaicin injection and in normal rats $(n=3)$. The specimens were homogenized in $0.5 \%$ hexadecyltrimethylammonium bromide (Sigma, St. Louis, MO) to temporarily neutralize enzyme activity. The samples subsequently underwent three cycles of freezing and thawing, followed by centrifugation to remove debris. A Spectramax 190 (Molecular Devices, Sunnyvale, CA) plate reader at a wavelength of $450 \mathrm{~nm}$ was used to read the changes in absorbance over time. Samples were reacted with hydrogen peroxide and $o$-dianisidine dihydrochloride at ambient temperature. Absorbance was measured every minute for up to $10 \mathrm{~min}$. $V_{\max }$ was used to determine the units of myeloperoxidase per gram of tissue from the standard curve. Samples were prepared in triplicate and averaged. Myeloperoxidase obtained from humans (Sigma) was used as a standard. The assay showed a $3 \%$ coefficient of variance between days and a limit of quantification of $0.07 \mathrm{U} / \mathrm{gm}$ tissue.

Behavioral testing. Animals were tested for withdrawal thresholds to mechanical stimuli (von Frey filaments) applied to the distal portion of the plantar aspect of the hindpaw (Sluka, 1997; Gopalkrishnan and Sluka, 2000). von Frey filaments with bending forces from 7 to $360 \mathrm{mN}$ were applied in a progressively increasing manner until the hindpaw was withdrawn or $360 \mathrm{mN}$ was reached. Each filament was applied twice. The filament of lowest bending force from which the animal withdrew was considered the mechanical withdrawal threshold of the hindpaw. After a response, the filaments above and below were tested to confirm the withdrawal threshold. The test-retest reliability of this method has been established previously (Gopalkrishnan and Sluka, 2000).

Animals were also tested for response to radiant heat applied to the plantar surface of the paw as described previously (Hargreaves et al., 1988). Briefly, animals were placed in clear plastic cubicles on an elevated glass plate and allowed to acclimate for 10-20 min before testing. The latency to withdrawal of the paw from radiant heat was measured. Five trials per time were averaged to give one number per animal. The test-retest reliability of this method has been established previously (Sluka et al., 1999).

Both mechanical withdrawal thresholds and response to radiant heat were applied to the caudal portion of the paw, which was outside the site of capsaicin injection in the skin, muscle, or joint. Thus, behavioral tests are interpreted as a measure of secondary hyperalgesia or allodynia (Sluka, 1997; Sluka and Willis, 1997).

Motor testing. Because $100 \mathrm{nmol}$ of PKA inhibitor (PKI) produced significant motor effects in five animals, rats administered the $60 \mathrm{nmol}$ dose of PKI $(n=4)$ were tested for motor effects with a Rota-Rod treadmill test and placing reflex test. Specifically, they were placed on a Rota-Rod treadmill (Ugo Basile Rota-Rod; Stoelting, Wood Dale, IL) rotating at a gradually increasing speed from 1 to $18 \mathrm{rpm}$ for $120 \mathrm{sec}$ and maintained for another $30 \mathrm{sec}$ at $18 \mathrm{rpm}$ (Sluka et al., 2001). For stable
Rota-Rod treadmill results, the rats were trained for $2 \mathrm{~d}$ before injection of drug. Three sessions of three trials each were performed at an intersession interval of $>2 \mathrm{hr}$ and an intertrial interval of $>5 \mathrm{~min}$. Performance time in minutes was recorded until the rat failed to stay on the Rota-Rod or reached $150 \mathrm{sec}$. The placing reflex was also evaluated after drug administration (Coderre and Van Empel, 1994; Zahn and Brennan, 1998). This test is performed by drawing the dorsum of the hindpaw across the edge of a table. The animal responds by lifting the paw onto the surface of the table. This was scored as 2, normal; 1 , delay of $1-2 \mathrm{sec}$; and $0,>2 \mathrm{sec}$. Motor function was assessed at baseline and 15 , 30,45 , and 60 min after administration of drug.

Intrathecal catheterization. Intrathecal catheters $(n=142 ; 32$ gauge polyurethane; $10 \mathrm{~cm}$ length; Recathco, Allison Park, PA) were placed 5-6 d before the first intramuscular injection of saline (Storkson et al., 1996; Pogatzki et al., 2000). In brief, animals were anesthetized with halothane $(2-5 \%)$, and a 23 gauge hypodermic needle was inserted into the intervertebral space between L5 and L6. A 32 gauge polyurethane catheter was inserted through the needle and advanced cranially unti $3.5-4.0 \mathrm{~cm}$ of the catheter was under the skin. The external portion of the catheter was secured to the muscle and fascia. The free end was subsequently inserted into polyethylene-10 tubing and tunneled to the cervical region.

Experimental design. In the first set of experiments, capsaicin was injected into the skin $(n=6)$, muscle $(n=13)$, or joint $(n=12)$. Behavioral tests were performed before the injection and hourly through $4,8,24$, and $48 \mathrm{hr}$ and 1 week and then weekly until the allodynia resolved. Responses to heat and mechanical stimuli were assessed at each time period.

The second series of experiments was designed to test the role of the cAMP pathway in the maintenance of the mechanical allodynia and heat hypoalgesia observed in the first experiment after injection of capsaicin into the muscle or joint. Behavioral tests were performed $24 \mathrm{hr}$ or 1 week after injection of capsaicin into the muscle or joint, and then 9-(tetrahydro-2' -furyl)adenine (SQ22536; blocks adenylate cyclase; 0.07$0.7 \mathrm{nmol} / 5 \mu \mathrm{l} ; n=51$; Biomol Research Laboratories Inc, Plymouth Meeting, PA), myristoylated PKI (14-22) amide (blocks PKA; 10-100 $\mathrm{nmol} / 5 \mu \mathrm{l} ; n=45$; Biomol Research Laboratories Inc), or vehicle $(n=$ 21) was injected intrathecally. Each dose was given to only one animal, and each group consisted of three to eight animals. Heat testing began 10 min after administration of drug and was subsequently performed at 5 min intervals for $30 \mathrm{~min}$. After the heat testing, 40-45 min after injection of drug, the mechanical withdrawal threshold was tested. We demonstrated previously that spinal application of either SQ22536 or PKI has no effect on normal behavioral responses (Sluka, 1997).

In the last series of experiments, the effects of spinal activation of the cAMP pathway on mechanical and heat sensitivity were assessed. 8-Bromo-cAMP (3-30 nmol $/ 5 \mu \mathrm{l} ; n=16)$ was injected intrathecally, and withdrawal latency to heat was measured for $30-45$ min after injection, followed by withdrawal threshold to mechanical stimulation at $\sim 35-45$ min after injection. A higher dose of 8-bromo-cAMP, $100 \mathrm{nmol} / 5 \mu \mathrm{l}$, was tested in three animals but produced agitation, making it difficult to test the animals, and thus was not tested further. The effect of pretreatment with PKI $(60 \mathrm{nmol} / 5 \mu \mathrm{l})$ on the effects of $30 \mathrm{nmol} / 5 \mu \mathrm{l}$ 8-bromo-cAMP was also tested $(n=4)$.

Statistical analysis. Differences between groups and across time for paw withdrawal latency to heat were analyzed with a one-way ANOVA. Post hoc testing was done as appropriate with a Tukey's test. Because there was no difference between normal tissue and the contralateral side, side-to-side differences were calculated for myeloperoxidase activity, and a one-way ANOVA was used to analyze differences between groups. Post hoc testing was done as appropriate with a Tukey's test. Kruskal-Wallis ANOVA was used to analyze differences in withdrawal threshold to mechanical stimuli between groups. Post hoc testing was done as appropriate with a sign test. Data were reported as the mean \pm SEM for paw withdrawal latency to heat and myeloperoxidase assay and as the median \pm 25 th and 75 th percentiles for mechanical withdrawal threshold.

\section{RESULTS}

\section{Behavioral effects of capsaicin}

As reported previously (Sluka, 1997), intradermal injection of capsaicin resulted in a decrease in the withdrawal threshold to mechanical stimuli by $30 \mathrm{~min}$ that lasted $\sim 3 \mathrm{hr}$ after injection (Fig. 1). The withdrawal threshold to mechanical stimuli returned to baseline threshold values by $4 \mathrm{hr}$ after injection. Similarly, the 

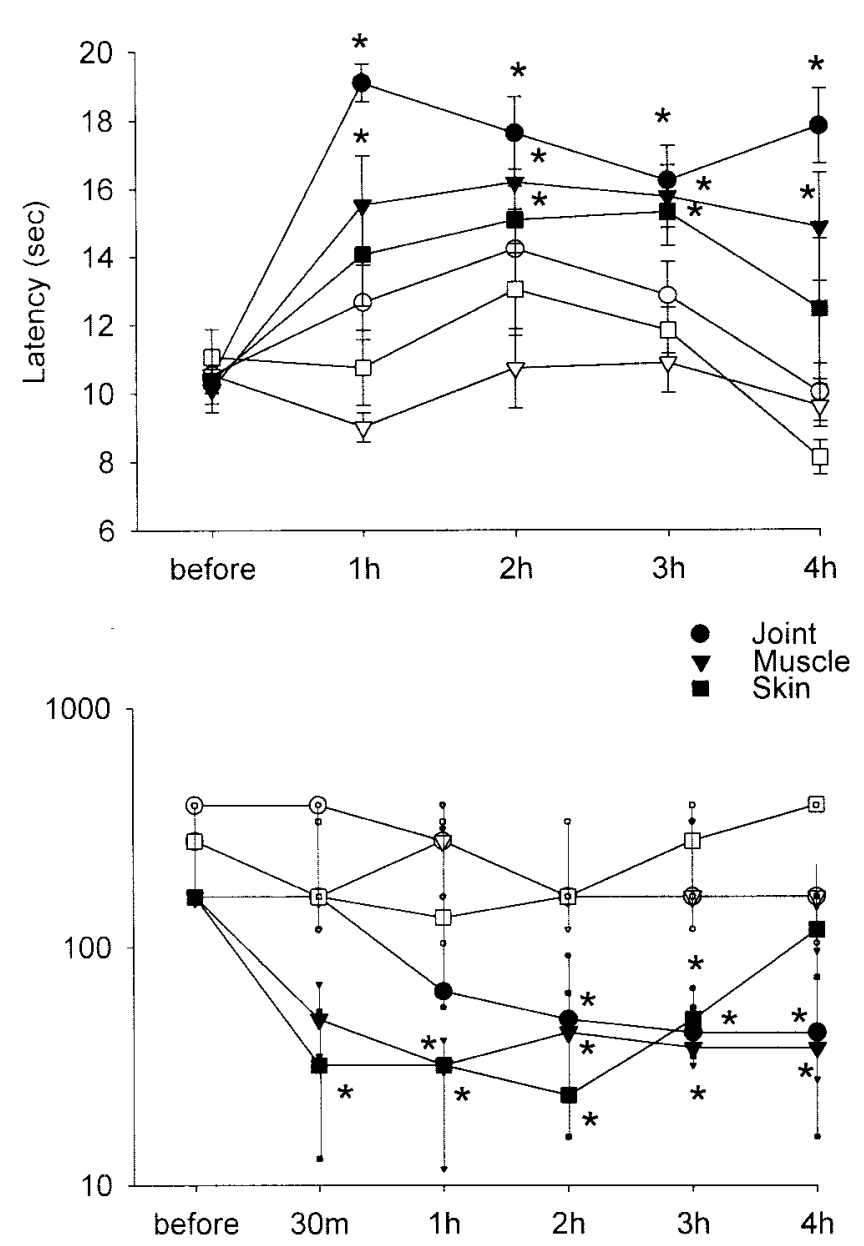

Figure 1. The paw withdrawal latency to heat (top) and withdrawal threshold to mechanical stimulation (bottom) are shown before and for 4 $\mathrm{hr}$ after injection of capsaicin into the skin (squares), muscle (triangles), or joint (circles) for the ipsilateral (closed symbols) or the contralateral (open symbols) side. Significant increases in withdrawal latency to heat ipsilaterally occur after injection of capsaicin into the skin, muscle, or joint. No changes are observed for the contralateral paw. ${ }^{*} p<0.05$, significantly different from before capsaicin injection.

withdrawal threshold to mechanical stimuli decreased by $30 \mathrm{~min}$ after injection into the plantar muscles of the paw (Fig. 1). However, it remained decreased through 1 week and resolved by 2 weeks after injection of capsaicin into the muscle. Furthermore, the mechanical withdrawal threshold decreased contralaterally 24 hr after capsaicin injection into the muscle and remained decreased through 1 week, returning to baseline by 2 weeks (Fig. 2). In contrast, capsaicin injection into the ankle joint showed a longer time to onset, with allodynia developing by $2 \mathrm{hr}$. This decrease in withdrawal threshold spread to the contralateral side after $24 \mathrm{hr}$ and lasted through 4 weeks (Fig. 2). Minimal limb guarding occurred within the first few hours after injection of capsaicin into the skin, muscle, or joint, and by $24 \mathrm{hr}$, no differences between ipsilateral and contralateral sides were observed.

The response to heat stimuli was surprising. Specifically, injection of capsaicin into the skin, plantar muscles, or ankle joint resulted in a significant increase in the latency to heat stimulation (Fig. 1). The increase after injection into the deep tissues, muscle, or joint was long lasting. This increase occurred only on the side that had received an injection and lasted through 2 weeks for injection into the muscles and 5 weeks for injection into the ankle joint. The contralateral side remained at baseline throughout the testing period.

\section{Assessment of inflammation}

Of the three tissues tested, the joint tissue had the highest baseline levels of myeloperoxidase activity. There were no significant differences between normal tissue and the contralateral side. For this reason, differences between the side injected with capsaicin and the contralateral side were analyzed. Capsaicin injected into the muscle or joint resulted in significant increases in myeloperoxidase activity 2 and $24 \mathrm{hr}$ after injection compared with tissue from animals without inflammation (Table 1). Significant increases in myeloperoxidase activity also occurred after capsaicin injection into the skin $24 \mathrm{hr}$ after injection but not at 2 hr or 1 week. There was no difference between tissue types $2 \mathrm{hr}$, $24 \mathrm{hr}$, or 1 week after inflammation, suggesting that a similar degree of inflammation was produced by a single injection of $0.2 \%$ capsaicin.

\section{Inhibition of adenylate cyclase}

SQ22536 was injected intrathecally into the spinal cord to assess the role of adenylate cyclase in the mechanical allodynia and heat hypoalgesia associated with capsaicin injection into the muscle or joint. Twenty-four hours and 1 week after injection of capsaicin, a bilateral decrease in withdrawal threshold to mechanical stimulation and an ipsilateral increase in withdrawal latency to heat stimulation occurred. Intrathecal injection of SQ22536, administered $24 \mathrm{hr}$ or 1 week after capsaicin injection, reversed the increased paw withdrawal latency significantly in a dosedependent manner compared with vehicle controls (Fig. 3). Furthermore, intrathecal injection of SQ22536 significantly reversed the decreased mechanical withdrawal threshold when administered $24 \mathrm{hr}$ after injection of capsaicin. Surprisingly, intrathecally administered SQ22536 had no effect on the decreased withdrawal threshold to mechanical stimuli induced by capsaicin when administered 1 week after injection of capsaicin into the joint (Fig. 4).

\section{Inhibition of PKA}

Intrathecal blockade of PKA with PKI resulted in a pattern similar to that observed by blockade of adenylate cyclase with SQ22536. Specifically, PKI dose-dependently reversed the increased latency to radiant heat when administered either $24 \mathrm{hr}$ or 1 week after injection of capsaicin (Fig. 3). The decreased withdrawal threshold to mechanical stimuli associated with capsaicin injection into the muscle or ankle joint was reversed when PKI was administered $24 \mathrm{hr}$ after injection of capsaicin but not when it was administered 1 week after injection of capsaicin (Fig. 4). Unexpectedly, the $100 \mathrm{nmol}$ dose of PKI resulted in significant motor deficits in five animals, and they were removed from the study. Motor testing of the $60 \mathrm{nmol}$ dose of PKI had no effect on the Rota-Rod treadmill test for up to $1 \mathrm{hr}$ after administration of drug, with rats staying on the treadmill for the full $150 \mathrm{sec}$. The placing reflex was normal in all four animals for up to $1 \mathrm{hr}$ after administration of $60 \mathrm{nmol}$ of PKI.

\section{Activation of cAMP}

Intrathecal injection of 8-bromo-cAMP resulted in a dosedependent increase in the withdrawal latency to radiant heat and a simultaneous decrease in the withdrawal threshold to mechanical stimuli (Fig. 5). The increase in withdrawal latency to heat and decrease in withdrawal threshold to mechanical stimuli were prevented by previous treatment with an intrathecal injection of $60 \mathrm{nmol}$ of PKI. 
Figure 2. Responses to mechanical and heat stimuli after injection of capsaicin into the ankle joint or plantar muscles of the foot for the ipsilateral (closed circles) and contralateral (open circles) paws. Significant bilateral decreases in withdrawal threshold to mechanical stimuli are evident through 6 weeks after injection into the ankle joint and 1 week after injection into the plantar muscles. The response to heat is elevated ipsilaterally for 6 weeks after injection into the ankle joint and 2 weeks after injection into the plantar muscles. ${ }^{*} p<0.05$, significantly different from baseline.
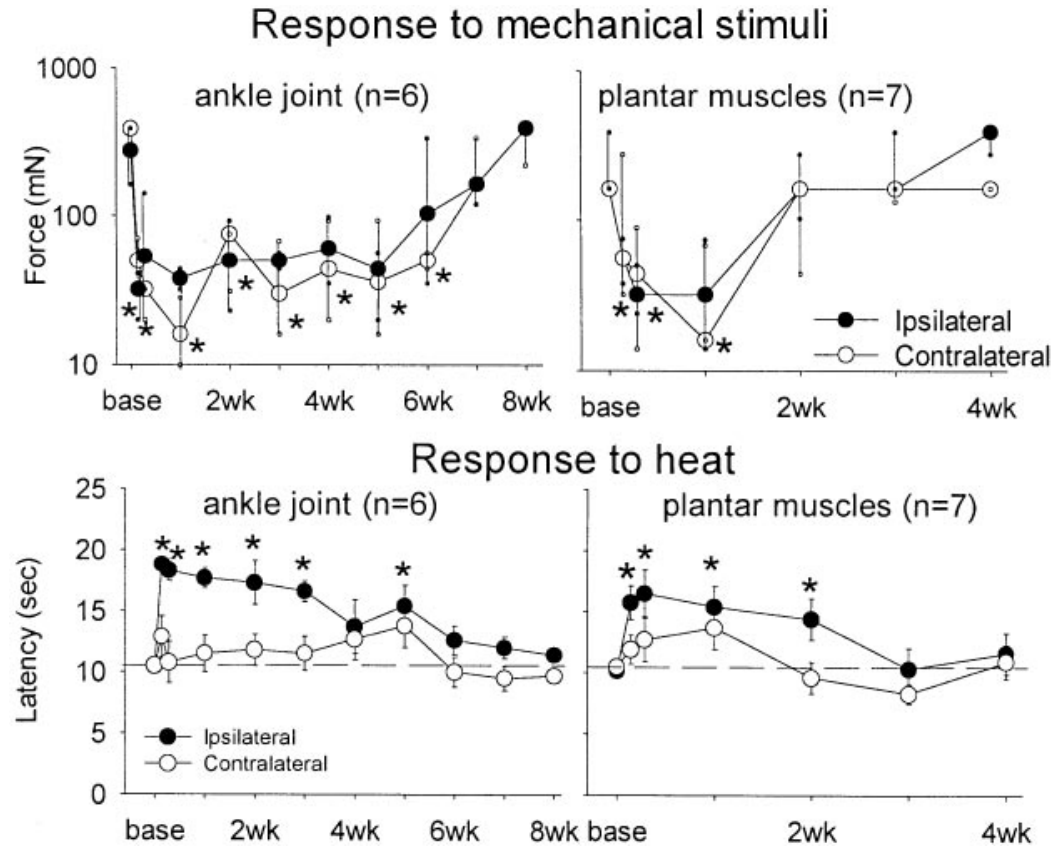

\begin{tabular}{lcclc}
\hline Table 1. Myeloperoxidase activity & & \\
& $\begin{array}{l}\text { Normal } \\
(n=3)\end{array}$ & $\begin{array}{l}\text { hr after } \\
\text { capsaicin } \\
(n=4)\end{array}$ & $\begin{array}{l}\text { 24 hr after } \\
\text { capsaicin } \\
(n=4)\end{array}$ & $\begin{array}{l}\text { 1 week after } \\
\text { capsaicin } \\
(n=4)\end{array}$ \\
\hline Skin & $0.08 \pm 0.01$ & $0.9 \pm 0.5$ & $3.3 \pm 0.7^{*}$ & $0 \pm 0.1$ \\
Muscle & $-0.11 \pm 0.5$ & $0.82 \pm 0.35^{*}$ & $6.6 \pm 0.2^{*}$ & $-0.04 \pm 0.01$ \\
Joint & $-0.46 \pm 0.5$ & $2.2 \pm 0.9^{*}$ & $1.9 \pm 1.3^{*}$ & $3.6 \pm 2.2$ \\
\hline
\end{tabular}

Left-right difference scores for myeloperoxidase activity in tissue taken from animals with and without inflammation. Data are mean \pm SEM in units per gram of tissue.

*Significantly different from normal.

\section{DISCUSSION}

\section{Differences between skin, muscle, and joint}

The present data show that intra-articular or intramuscular injection of capsaicin results in a long-lasting mechanical allodynia and heat hypoalgesia of the skin. Furthermore, the mechanical allodynia spreads to the contralateral paw $\sim 24 \mathrm{hr}$ after capsaicin injection into muscle or joint. The allodynia resolves more quickly if injected into the muscle compared with the ankle joint. Although the ankle joint, plantar muscle, and plantar aspect of the skin are supplied by branches of the tibial nerve (Greene, 1963), different central anatomic pathways and/or different biochemical mediators could result in a different pattern of response. Dorsal root ganglia neurons innervating muscle and joint have less isolectin B4 and somatostatin and more calcitonin generelated peptide and substance $\mathrm{P}$ than dorsal root ganglia neurons innervating cutaneous tissue (O'Brien et al., 1989; Plenderleith and Snow, 1993). The central projections from neurons innervating muscle and joint are predominately to lamina I and deeper dorsal horn, whereas those from cutaneous tissue have a dense projection to lamina II (Craig et al., 1988; Mense and Craig, 1988; Mense, 1993; Schaible and Grubb, 1993). Formalin injected into skin increases c-Fos expression throughout laminas I-V, but when it is injected into muscle, there is no labeling in lamina II (Ohtori et al., 2000). Furthermore, C-fiber stimulation of a muscle nerve produces a longer-lasting increase in the flexion reflex compared
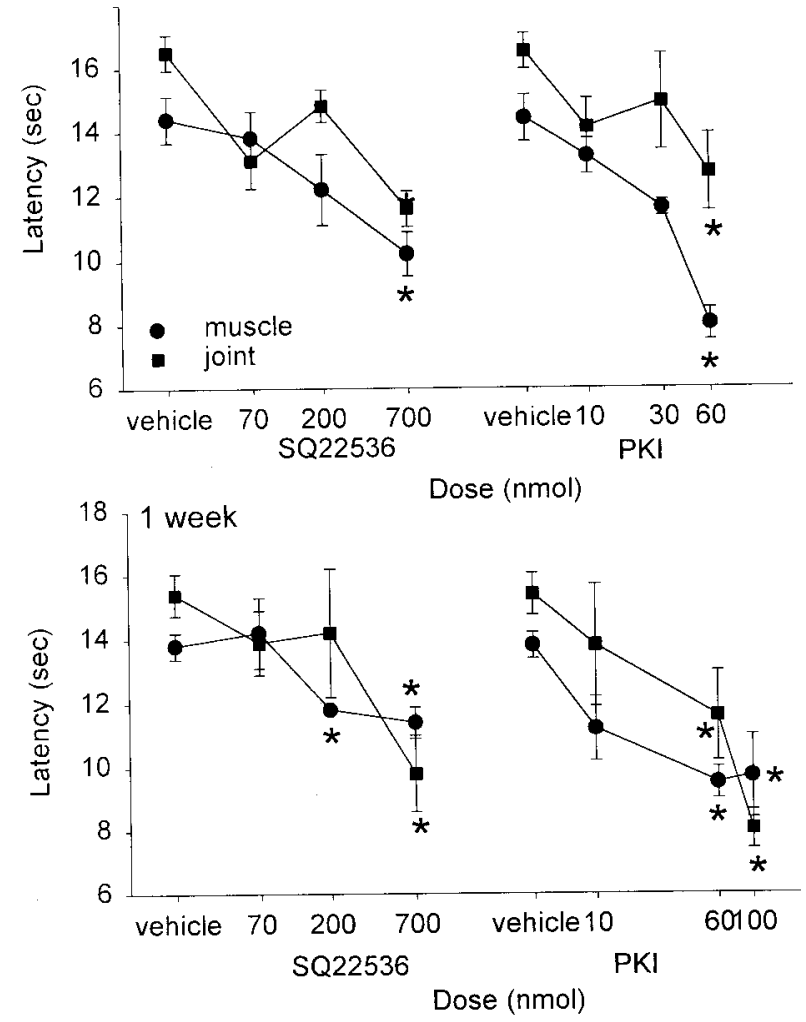

Figure 3. Withdrawal latency to heat ipsilaterally after spinal blockade with SQ22536 or PKI at $24 \mathrm{hr}$ (top) or 1 week (bottom). A dose-dependent reduction in the capsaicin-induced increased withdrawal latency to heat is observed after spinal blockade of adenylate cyclase with SQ22536 or PKA with PKI. ${ }^{*} p<0.05$, significantly different from vehicle control.

with C-fiber stimulation of a cutaneous nerve (Wall and Woolf, 1984). In human subjects, painful intramuscular stimulation is rated as more unpleasant than painful cutaneous stimulation (Svensson et al., 1997). Capsaicin injected into muscle of human subjects produces longer-lasting pain, and referred pain is more 

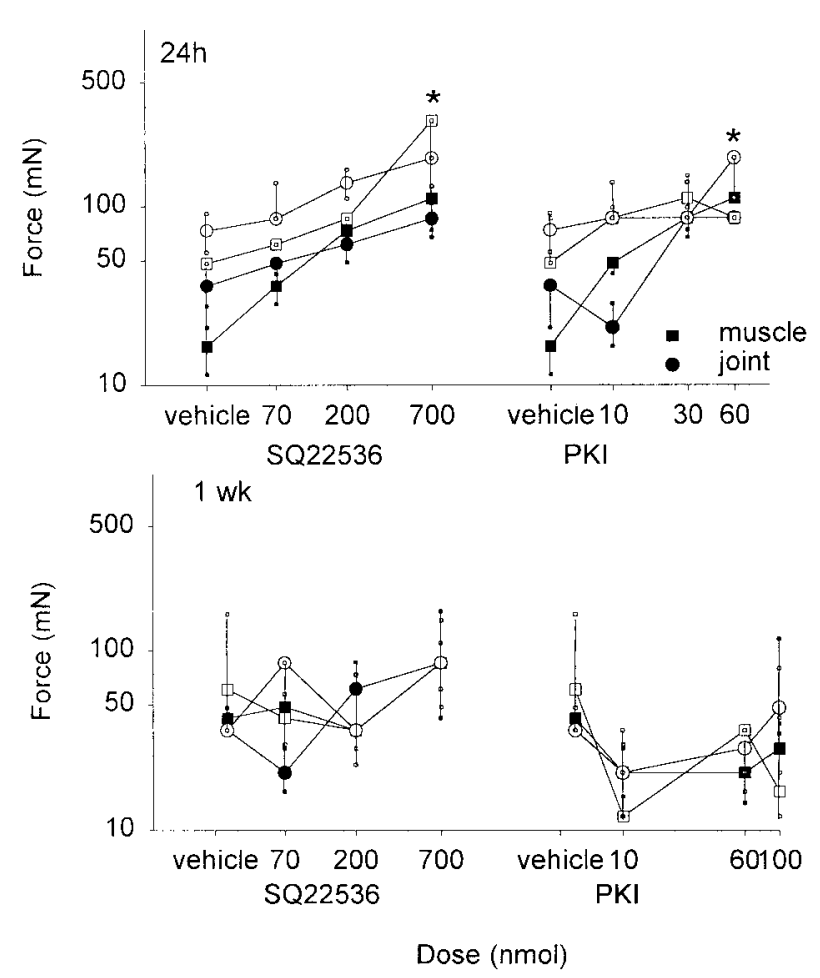

Figure 4. Withdrawal threshold to mechanical stimuli bilaterally $24 \mathrm{hr}$ and 1 week after injection of capsaicin. After spinal blockade with SQ22536 or PKI at $24 \mathrm{hr}$ (top), there is a dose-dependent inhibition of the capsaicin-induced decrease in the withdrawal threshold to mechanical stimuli for animals injected with capsaicin into the muscle (squares) or joint (circles). No effect of SQ22536 or PKI occurred when they were administered 1 week after injection of capsaicin for either the ipsilateral or contralateral paw (bottom). The contralateral side is depicted with open symbols, and the ipsilateral side is depicted with closed symbols. * $p<0.05$, significantly different from vehicle control.

frequent (Witting et al., 2000). Thus, biochemical, anatomic, and physiological differences support the hypothesis that injury to muscle and joint results in distinctly different behavioral responses compared with skin.

Alternatively, capsaicin injection into muscle or joint may produce a more severe neurogenic inflammation than injection into skin. However, assessment of neutrophilic activity after capsaicin injection shows that the amount of inflammation is similar between muscle, skin, and joint tissues. Peak activity occurs $24 \mathrm{hr}$ after injection for all three tissues, a time in which there was no allodynia from injection into skin. These data support differential processing of nociceptive information from muscle and joint compared with skin. Measurement of myeloperoxidase activity as a measure of neutrophil infiltration is used extensively to measure acute inflammation for periods up to 1 week after induction of inflammation in several animal models (Recio et al., 2000; Reifen et al., 2001). It should also be noted that, although it was our intention to inject capsaicin into the muscle tissue of the paw, it could also have affected tendons that course through the muscle layers in the foot and ligaments that surround the small joints of the foot. However, visually, the muscle of the paw was inflamed and the skin was not red, suggesting that the injection into the plantar muscles did affect predominantly deep and not cutaneous tissues.

Finally, repeated behavioral testing could result in a learning paradigm that caused the animals to withdraw at a lower bending
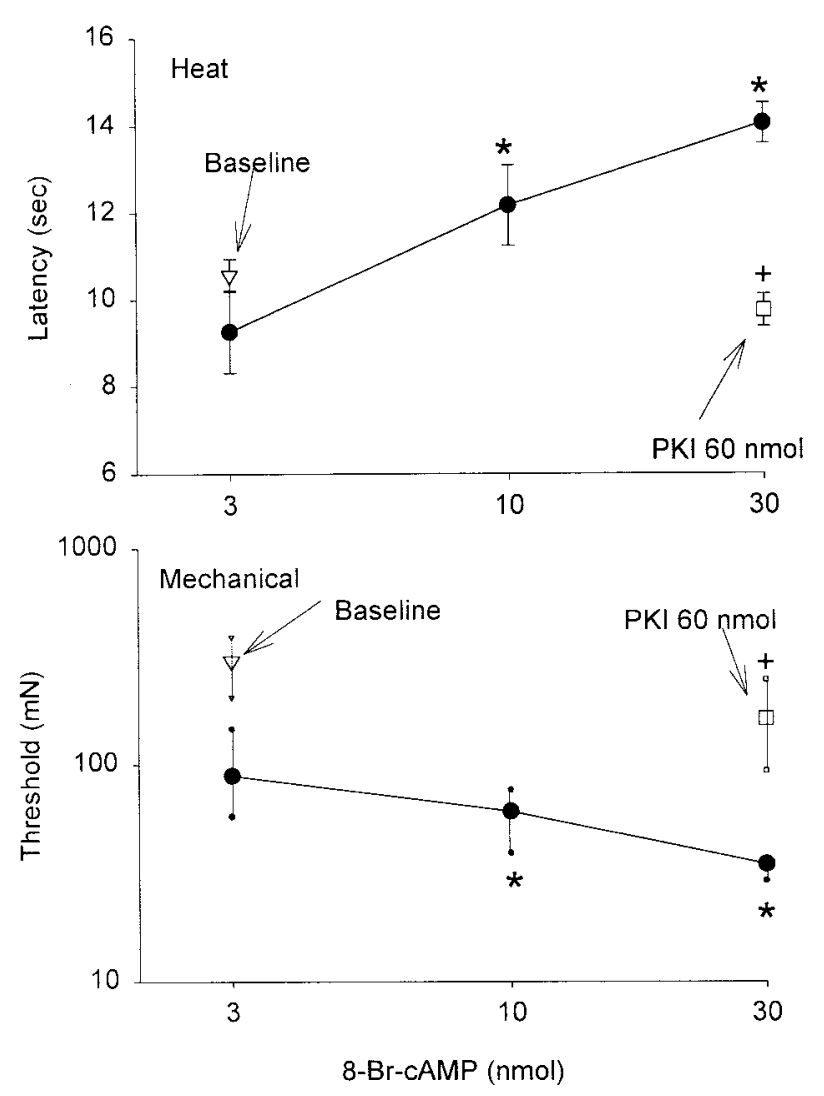

Figure 5. Intrathecal administration of 8-bromo-cAMP resulted in a simultaneous increase in the withdrawal latency to heat and a decrease in the withdrawal threshold to mechanical stimuli compared with baseline. Pretreatment with $60 \mathrm{nmol}$ of PKI prevented the increase in withdrawal latency to heat and the decrease in withdrawal threshold to mechanical stimuli produced by $30 \mathrm{nmol}$ of 8 -bromo-cAMP. ${ }^{*} p<0.05$, significantly different from baseline; ${ }^{+} p<0.05$, significantly different from $30 \mathrm{nmol}$ of 8-bromo-cAMP.

force or with a faster latency to heat, and it would thus appear that the animals were hyperalgesic to mechanical stimuli and/or heat stimuli. Previous data show that the responses of the animals did not change with repeated testing to mechanical and heat stimuli over a 6 week time course (Sluka et al., 2001). Furthermore, the increase in heat response, with a simultaneous decrease in mechanical response and different time course of behavioral changes after injection into muscle or joint tissue, argues against a learned behavior.

\section{Activation of the cAMP pathway and mechanical allodynia}

A time-dependent effect on mechanical allodynia was observed in the present study such that when adenylate cyclase or PKA is inhibited $24 \mathrm{hr}$ after capsaicin injection, allodynia is reversed, but when it is inhibited 1 week after capsaicin injection, there is no effect. Similarly, mice lacking the type I regulatory subunit of PKA showed attenuated nocifensive behaviors to the shortlasting stimuli formalin and prostaglandin $\mathrm{E}_{2}$ but still developed hyperalgesia to a more chronic injury, neuropathic pain (Malmberg et al., 1997a). Alternatively, mice lacking PKC $\gamma$ show a deficit in hyperalgesia associated with neuropathic pain but no deficit associated with the first phase of the formalin test, which is thought to represent acute pain (Malmberg et al., 1997b). However, the second phase of the formalin test was reduced in mice 
lacking PKC $\gamma$ (Malmberg et al., 1997b). The later stages of mechanical allodynia induced by intramuscular or intra-articular injection of capsaicin could thus be maintained by PKC.

Previous studies show that the mechanical allodynia that occurs after intradermal injection of capsaicin is reversed by spinal administration of the same inhibitors of PKA and adenylate cyclase (Sluka, 1997; Sluka and Willis, 1997). Furthermore, the present study shows that 8 -bromo-cAMP produces mechanical allodynia in a dose-dependent manner and agrees with other studies (Sluka, 1997; Sluka and Willis, 1997; Dolan and Nolan, 2001). However, a low dose of 8-bromo-cAMP produces mechanical hypoalgesia in sheep, but increasing the dose produces mechanical hyperalgesia (Dolan and Nolan, 2001).

Activation of adenylate cyclases in neurons can occur through neurotransmitter-receptor interactions that are linked to stimulatory G-proteins. Alternatively, three (of nine) adenylate cyclase isoforms (AC1, AC3, and AC8) are calcium-calmodulin regulated (Xia and Storm, 1997). Activation of adenylate cyclase then converts ATP to cAMP, which results in activation of PKA. Activation of protein kinases can result in neuroplasticity through phosphorylation of a variety of substrates including ion channels, neurotransmitter receptors, and transcription factors. PKA phosphorylates calcium channels, glutamate receptors, and the cAMP response element-binding protein (Dash et al., 1991; Blackstone et al., 1995; Hell et al., 1995). Indeed, phosphorylation of the PKA site of the NR1 subunit of NMDA glutamate receptors is increased after intradermal injection of capsaicin (Zou et al., 2000). Injection of PKA enhances dorsal horn neuron responsiveness to glutamate (Cerne et al., 1992, 1993), whereas blockade of PKA reduces capsaicin-induced sensitization of spinothalamic tract cells (Sluka et al., 1997). Phosphorylation of the cAMP response element-binding protein enhances gene transcription of cell proteins (Dash et al., 1991; Shaywitz and Greenberg, 1999) and increases after carrageenan inflammation (Messersmith et al., 1996), formalin injection (Ji and Rupp, 1997; Anderson and Seybold, 2000), sciatic nerve stimulation at C-fiber strength (Ji et al., 2000), and neuropathic pain (Ma and Quirion, 2001).

The role of the cAMP pathway in neuroplasticity has been studied extensively with respect to long-term potentiation (LTP) and learning in the hippocampus and parallels the timedependent activation of the cAMP pathway observed in the present study. Specifically, PKA activity increases in the early phases of spatial learning tasks (Vazquez et al., 2000). PKC appears to be important in the maintenance as well as the initiation of LTP and learning (Sweatt, 1999; Vazquez et al., 2000). In learning and memory behavioral tasks, mice deficient in the calcium-dependent adenylate cyclases AC1 and AC8 show a selective deficit in hippocampus-dependent learning and LTP (Wong et al., 1999). Thus, the mechanical allodynia observed in the present study shows an activation pattern of the cAMP pathway similar to that observed in LTP and memory. Specifically, activation of the CAMP second messenger pathway is necessary during the early phase of development but not in the later maintenance phase. Furthermore, on the basis of these similarities and previous data with PKC $\gamma$ knock-out mice (Malmberg et al., 1997a), it is possible that PKC is important in the maintenance phase of mechanical allodynia.

\section{Activation of the cAMP pathway and heat hypoalgesia}

The heat hypoalgesia that occurs after capsaicin injection could be related to damage to peripheral nerves, changes in peripheral nociceptors, or changes in the CNS. The reversal of heat hypoal- gesia by spinal blockade of adenylate cyclase or PKA suggests that this hypoalgesia is mediated by plastic changes in the CNS rather than by damage to peripheral nerves. The effects may be related to changes in spinothalamic tract neurons and not to modulation of neurotransmitter release. Previous studies show a decreased responsiveness of spinothalamic tract cells to heat stimuli after intradermal injection of capsaicin (Sluka et al., 1997; Dougherty et al., 1998) that is reversed by spinal blockade of PKA (Sluka et al., 1997). PKA has no effect on increased excitatory amino acid release that occurs after capsaicin injection (Sluka and Willis, 1998). More recently, Liu et al. (2001) demonstrated that capsaicin desensitizes primary afferent nociceptors by inhibiting sodium channel conductance and that this inhibition is mediated by cAMP. Vanilloid 1 receptors are activated by capsaicin, are thought to be heat sensors, are found on nociceptors, and desensitize after capsaicin injection. It is possible that this desensitization also occurs on central terminals of primary afferents and that the effects of spinal inhibition of adenylate cyclase and PKA are to reverse cAMP-mediated desensitization.

\section{Summary}

In summary, this study showed that activation of the cAMP pathway in the spinal cord results in divergent behavioral signs depending on the modality of stimulus tested (i.e., mechanical or heat), suggesting that processing of mechanical and heat responsiveness to tissue injury involves unique processes. Furthermore, activation of the cAMP pathway is time-dependent with respect to maintenance of mechanical allodynia and appears to be necessary in the early phases of central sensitization but not in later phases. Finally, deep tissue injury results in longer-lasting allodynia compared with the same stimulus applied to the skin, supporting the theory that nociceptive processing from deep tissue injury is distinct from nociceptive processing from cutaneous tissue injury.

\section{REFERENCES}

Anderson LE, Seybold VS (2000) Phosphorylated cAMP response element binding protein increases in neurokinin-1 receptorimmunoreactive neurons in rat spinal cord in response to formalininduced nociception. Neurosci Lett 283:29-32.

Blackstone C, Murphy TH, Moss SJ, Baraban JM, Huganir RL (1995) Cyclic AMP and synaptic activity dependent phosphorylation of AMPA preferring glutamate receptors. J Neurosci 14:7585-7593.

Cerne R, Jiang M, Randic M (1992). Cyclic adenosine 3' $5^{\prime}$ monophosphate potentiates excitatory amino acid and synaptic responses of rat spinal dorsal horn neurons. Brain Res 596:111-123.

Cerne R, Rusin KI, Randic M (1993) Enhancement of the N-methyl-Daspartate response in spinal dorsal horn neurons by cAMP-dependent protein kinase. Neurosci Lett 161:124-128.

Coderre TJ, Van Empel I (1994) The utility of excitatory amino acid (EAA) antagonists as analgesic agents. I. Comparison of the antinociceptive activity of various classes of EAA antagonists in mechanical, thermal, and chemical nociceptive tests. Pain 59:345-352.

Craig AD, Hepplemann B, Schaible H-G (1988) The projection of the medial and posterior articular nerves of the cat's knee to the spinal cord. J Comp Neurol 276:279-288.

Dash PK, Karl KA, Colicos MA, Prywes R, Kandel ER (1991) cAMP response element-binding protein is activated by $\mathrm{Ca}^{2+} /$ calmodulin as well as cAMP-dependent protein kinase. Proc Natl Acad Sci USA 88:5061-5065.

Desser RK, Himmelhoch SR, Evans WH, Januska M, Mage M, Shelton E (1972) Heterophil and eosinophil peroxidase. Arch Biochem Biophys 148:452-465.

Dolan S, Nolan AM (2001) Biphasic modulation of nociceptive processing by the cyclic AMP-protein kinase A signalling pathway in sheep spinal cord. Neurosci Lett 309:157-160.

Dougherty PM, Willis WD, Lenz FA (1998) Transient inhibition of responses to thermal stimuli of spinal sensory tract neurons in monkeys during sensitization by intradermal capsaicin. Pain 77:129-136.

Gopalkrishnan P, Sluka KA (2000) Effect of varying frequency, intensity and pulse duration of TENS on primary hyperalgesia in inflamed rats. Arch Phys Med Rehabil 81:984-990. 
Greene EC (1963) Anatomy of the Rat, Vol XXVII. New York: Hafner.

Hargreaves K, Dubner R, Brown F, Flores C, Joris J (1988) A new, sensitive method for measuring thermal nociception in cutaneous hyperalgesia. Pain 32:77-88.

Hell JW, Yokoyama CT, Breeze LJ, Chavkin C, Catterall WA (1995) Phosphorylation of presynaptic and postsynaptic calcium channels by cAMP dependent protein kinase in hippocampal neurons. EMBO J 14:3036-3044.

Ji RR, Rupp F (1997) Phosphorylation of transcription factor CREB in rat spinal cord after formalin-induced hyperalgesia: relationship to c-fos induction. J Neurosci 17:1776-1785.

Ji RR, Brenner GJ, Schmoll R, Baba H, Woolf CJ (2000) Phosphorylation of transcription factor CREB in nociceptive neurons after noxious stimulation. Proceedings of the Ninth World Congress on Pain 16:1776-1785.

Kellgren JH (1938) Observations on referred pain arising from muscle. Clin Sci 3:175-190.

Lamotte RH, Shain CN, Simone DA, Tsai EFP (1991) Neurogenic hyperalgesia: psychophysical studies of underlying mechanisms. J Neurophysiol 66:190-211.

Liu L, Oortgiesen M, Li L, Simon SA (2001) Capsaicin inhibits activation of voltage-gated sodium currents in capsaicin-sensitive trigeminal ganglion neurons. J Neurophysiol 85:745-758.

Ma W, Quirion R (2001) Increased phosphorylation of cyclic AMP response element-binding protein (CREB) in the superficial dorsal horn neurons following partial sciatic nerve ligation. Pain 93:295-301.

Malmberg AB, Brandon EP, Idzerda RL, Liu H, McKnight GS, Basbaum AI (1997a) Diminished inflammation and nociceptive pain with preservation of neuropathic pain in mice with a targeted mutation of the type I regulatory subunit of cAMP-dependent protein kinase. J Neurosci 17:7462-7470.

Malmberg AB, Chen C, Tonegawa S, Basbaum AI (1997b) Preserved acute pain and reduced neuropathic pain in mice lacking PKCgamma. Science 278:279-283.

Marchettini P, Cline M, Ochoa J (1990) Innervation territories for touch and pain afferents of single fascicles of the human ulnar nerve. Brain 113:1491-1500.

Marchettini P, Simone DA, Caputi G, Ochoa JL (1996) Pain from excitation of identified muscle nociceptors in humans. Brain Res 75:109-116.

Mense S (1993) Nociception from skeletal muscle in relation to clinical muscle pain. Pain 54:241-289.

Mense S, Craig AD (1988) Spinal and supraspinal terminations of primary afferent fibers from the gastrocnemius-soleus muscle in the cat. Neuroscience 26:1023-1035.

Messersmith DJ, Kim DJ, Gu J, Dubner R, Iadarola MJ (1996) c-Jun activation of the DYNCRE3 site in the prodynorphin promoter. Brain Res Mol Brain Res 40:15-21.

O'Brien C, Woolf CJ, Fitzgerald M, Lindsay RM, Molander C (1989) Differences in the chemical expression of rat primary afferent neurons which innervate skin, muscle or joint. Neuroscience 32:493-502.

Ochoa J, Torebjork E (1983) Sensations by intraneuronal microstimulation of single mechanoreceptor units innervating the human hand. J Physiol (Lond) 73:633-654.

Ochoa J, Torebjork E (1989) Sensations evoked by intraneural microstimulation of $\mathrm{C}$ nociceptor fibres in human skin nerves. J Physiol (Lond) 415:583-599.

Ohtori S, Takahashi K, Chiba T, Takahashi Y, Yamagata M, Sameda H, Moriya $\mathrm{H}(2000)$ Fos expression in the rat brain and spinal cord evoked by noxious stimulation to low back muscle and skin. Spine 25:2425-2430.

Plenderleith MB, Snow PJ (1993) The plant lectin Bandeiraea simplicifolia I-B4 identifies a subpopulation of small diameter primary sensory neurones which innervate the skin in the rat. Neurosci Lett 159:17-20.

Pogatzki EM, Zahn PK, Brennan TJ (2000) Effect of pretreatment with intrathecal excitatory amino acid receptor antagonists on the development of pain behavior caused by plantar incision. Anesthesiology 93:489-496.

Raja SN, Campbell JN, Meyer RA (1984) Evidence for different mechanisms of primary and secondary hyperalgesia following heat injury to the glaborous skin. Brain 107:1179-1188.

Recio MC, Giner RM, Uriburu L, Manez S, Cerda M, DelaFuents JR,
Rios JL (2000) In vivo activity of pseudoguaianolide sesquiterpene lactones in acute and chronic inflammation. Life Sci 66:2509-2518.

Reifen R, Nur T, Matas Z, Halpern Z (2001) Lycopene supplementation attenuates the inflammatory status of colitis in a rat model. Int J Vitam Nutr Res 71:347-351.

Schaible H-G, Grubb BD (1993) Afferent and spinal mechanisms of joint pain. Pain 55:5-54.

Shaywitz AJ, Greenberg ME (1999) CREB: a stimulus-induced transcription factor activated by a diverse array of extracellular signals. Annu Rev Biochem 68:821-861.

Simone DA, Baumann TK, Lamotte RH (1989) Dose dependent pain and mechanical hyperalgesia in humans after intradermal injection of capsaicin. Pain 38:99-107.

Simone DA, Sorkin LS, Oh U, Chung JM, Owens C, Lamotte RH, Willis WD (1991) Neurogenic hyperalgesia: central neural correlates in responses of spinothalamic tract neurons. J Neurophysiol 66:228-246.

Simone DA, Marchettini P, Caputi G, Ochoa JL (1994) Identification of muscle afferents subserving sensation of deep pain in humans. J Neurophysiol 72:883-889.

Sluka KA (1997) The role of the cyclic AMP transduction cascade in mechanical allodynia and hyperalgesia induced by intradermal injection of capsaicin in rats. Br J Pharmacol 122:1165-1173.

Sluka KA (2000) Development and time course of secondary heat and mechanical hyperalgesia following capsaicin or carrageenan injection of muscle, joint or skin. Soc Neurosci Abstr 26:2192.

Sluka KA, Willis WD (1997) The effects of G-protein and protein kinase inhibitors on the behavioral responses of rats to intradermal injection of capsaicin. Pain 71:165-178.

Sluka KA, Willis WD (1998) Increased release of excitatory amino acids following intradermal injection of capsaicin is reduced by a protein kinase G inhibitor. Brain Res 798:281-286.

Sluka KA, Rees H, Chen PS, Tsuruoka M, Willis WD (1997) Inhibitors of G-proteins and protein kinases reduce the sensitization of spinothalamic tract neurons following intradermal injection of capsaicin in the primate. Exp Brain Res 115:15-24.

Sluka KA, Christy MR, Peterson WL, Rudd SL, Troy SM (1999) Reduction of pain-related behaviors with either cold or heat treatment in an animal model of acute arthritis. Arch Phys Med Rehab 80:313-317.

Sluka KA, Kalra A, Moore SA (2001) Unilateral intramuscular injections of acidic saline produce a bilateral, long-lasting hyperalgesia. Muscle Nerve 24:37-46.

Storkson RV, Kjorsvik A, Tjolsen A, Hole K (1996) Lumbar catheterization of the spinal subarachnoid space in the rat. J Neurosci Methods 65:167-172.

Svensson P, Beydoun A, Morrow TJ, Casey KL (1997) Human intramuscular and cutaneous pain: psychophysical comparisons. Exp Brain Res 114:390-392.

Sweatt JD (1999) Toward a molecular explanation for long-term potentiation. Learn Mem 6:399-416.

Vazquez SI, Vazquez A, Pena DO (2000) Different hippocampal activity profiles for PKA and PKC in spatial discrimination learning. Behav Neurosci 114:1109-1118.

Wall PD, Woolf CJ (1984) Muscle but not cutaneous C-afferent input produces prolonged increases in the excitability of the flexion reflex in the rat. J Physiol (Lond) 356:443-458.

Willis WD, Coggeshall RE (1991) Sensory mechanisms of the spinal cord. New York: Plenum.

Witting N, Svensson P, Gottrup H, Arendt-Nielsen L, Jensen TS (2000) Intramuscular and intradermal injection of capsaicin: a comparison of local and referred pain. Pain 84:407-412.

Wong ST, Athos J, Figueroa XA, Pineda VV, Schaefer ML, Chavkin CC, Muglia LJ, Storm DR (1999) Calcium-stimulated adenylyl cyclase activity is critical for hippocampus-dependent long-term memory and late phase LTP. Neuron 23:787-798.

Xia Z, Storm DR (1997) Calmodulin-regulated adenylyl cyclases and neuromodulation. Curr Opin Neurobiol 7:391-396.

Zahn PK, Brennan TJ (1998) Lack of effect of intrathecally administered $N$-methyl-D-aspartate receptor antagonists in a rat model of postoperative pain. Anesthesiology 88:143-156.

Zou X, Lin Q, Willis WD (2000) Enhanced phosphorylation of NMDA receptor 1 subunits in spinal cord dorsal horn and spinothalamic tract neurons after intradermal injection of capsaicin in rats. J Neurosci 20:6989-6997. 